\title{
Effect of Contralateral Hip Adduction on Activity of Lumbar Stabilizers and Pelvic Lateral Tilting During Hip Abduction in Side-Lying
}

\author{
Hyo-uen Kim ${ }^{1}$, MSc, PT, Oh-yun Kwon ${ }^{2,3}$, PhD, PT, Chung-hwi $\mathrm{Yi}^{2,3}$, PhD, PT, \\ Heon-seock Cynn ${ }^{2,3}, \mathrm{PhD}, \mathrm{PT}$, Houng-sik Choi ${ }^{4}, \mathrm{PhD}, \mathrm{PT}$ \\ ${ }^{1}$ Dept. of Health Care Center, Samsung Electronics, Kangbuk Samsung Hospital \\ ${ }^{2}$ Dept. of Physical Therapy, College of Health Science, Yonsei University \\ ${ }^{3}$ Dept. of Ergonomic Therapy, The Graduate School of Health and Environment, Yonsei University \\ ${ }^{4}$ Dept. of Physical Therapy, Hanseo University
}

\begin{abstract}
The purpose of this study was to determine the effect of contralateral hip adduction (CHA) on the muscle activity of lumbar stabilizers and the angle of pelvic lateral tilting during hip abduction in side lying. Twenty healthy male subjects with no medical history of lower extremity or lumbar spine disorders were recruited for the study. Subjects randomly performed preferred hip abduction (PHA) and hip abduction with contralateral hip adduction in side lying. The muscle activities of the dominant side rectus abdominis, external oblique, internal oblique, quadratus lumborum, gluteus medius, and non-dominant side hip adductor longus were measured during PHA and CHA by using a surface electromyography (EMG) system. Pelvic lateral tilting motion was measured by using a three-dimensional motion analysis system. Data on EMG and pelvic motion were collected at the same time during PHA and CHA. A paired t-test was used to compare EMG activity and the angle of pelvic lateral tilting in the two exercises. The study found that the EMG activities of all muscles were more increased significantly in CHA than PHA condition. The angle of pelvic lateral tilting was more decreased significantly in CHA than PHA condition. These findings suggest that CHA could be recommended as a hip abduction exercise for activating lumbar stabilizers and decreasing compensatory pelvic lateral tilting motion.
\end{abstract}

Key Words: Electromyography; Hip adduction; Lumbar stabilizers; Pelvic lateral tilting.

\section{Introduction}

Increasing lumbar stability is considered an effective method to prevent lumbar musculoskeletal disease and improve lumbar function. Lumbar stability is maintained through the isometric contraction of the lumbar and abdominal muscles during limb movement (Kisner and Colby, 2002). Previous studies have compared decreased and delayed activity of lumbar stabilizers during limb movement in patients with low back pain and those without low back pain (Hodge and Richardson, 1997; Sahrmann, 2002). Decreased lumbar stability during limb movement leads to compensatory movement. Excessive compen- satory movement causes micro trauma which when repeated, leads to lumbar dysfunction (Sahrmann, 2002).

If lumbar stability is decreased during hip abduction in side lying, pelvic lateral tilting can appear as a compensatory movement (Norris, 1995). It is normal pattern involves $40^{\circ}$ hip abduction with no hip flexion, external or internal rotation, hip elevation, or pelvic rotation (Janda, 1996). Hip abduction in side lying is an exercise that is commonly used to improve gait and balance ability (Judge et al, 1993; Sashika et al, 1996) and to evaluate gait patterns (Krebs et al, 1991). While many studies have investigated lumbar stabilization in a standing or supine

Corresponding author: Oh-yun Kwon kwonoy@yonsei.ac.kr 
lying position, few have investigated in a side lying position (Hodges and Richardson, 1999; Jull et al, 1993).

During hip abduction, the contraction of contralateral hip adduction (CHA) can stabilize the lumbar region (Lee, 1999). According to Lee (1999), the hip abductor and the contralateral hip adductor muscles are related closely in the kinematic chain and can exert forces and $\mathrm{co}^{-}$contract or $\mathrm{co}^{-}$-release to optimize the function of the pelvis. In addition, enough force is exerted by the contralateral hip adductor, it can maintain pelvic stability against the force of the hip abductor (Root and Spero, 1981). However, no existing study has reported on the role of CHA with regard to hip abduction in lumbar stability in side lying. Recently, methods of increasing lumbar stability and decreasing compensatory movement through internal fixation involving muscle contraction have been actively investigated (Cynn, 2006). If it is possible for individuals to increase their lumbar stability and decrease compensatory movement themselves, it would be a helpful means of decreasing the risk of low back pain during hip abduction exercises in side lying.

With this in mind, this study examined the effects of CHA on the activity of the lumbar stabilizers and on pelvic lateral tilting during hip abduction in side lying. It hypothesized that the activity of the lumbar stabilizers would be increased and that pelvic lateral tilting would be decreased in hip abduction with CHA as compared with preferred hip abduction (PHA).

\section{Methods}

\section{Subjects}

Twenty healthy male subjects from Yonsei University participated. The subjects were excluded if they had past or present neurological, musculoskeletal, cardiopulmonary disease, low back pain, knee pain, hip joint contracture, or gluteus medius strength below the grade of "good" in manual mus-
Table 1. Characteristics of the subjects

$(\mathrm{N}=20)$

\begin{tabular}{cc}
\hline \hline Parameter & Mean \pm SD \\
\hline Age $(\mathrm{yr})$ & $21.8 \pm 2.8$ \\
Body mass $(\mathrm{kg})$ & $71.9 \pm 10.8$ \\
Height $(\mathrm{cm})$ & $173.3 \pm 4.1$ \\
\hline
\end{tabular}

cle test. Dominant leg was identified as the leg used to kick a ball (Hoffman and Payne, 1995). All subjects were right-leg dominant. Prior to the study, ethics approval was obtained from Yonsei University. All subjects were informed about the purpose and procedures of the study and signed a written informed consent form. The demographic characteristics of the subjects are presented in Table 1.

\section{Instruments}

\section{Surface electromyography}

To measure muscle activity of the lumbar stabilizers, the Noraxon TeleMyo 2400T (Noraxon Inc., Scottsdale, AZ, USA) was used. The participant's skin was shaved, rubbed with sand-paper, and cleaned with alcohol. Pairs of surface electrodes were placed at an inter-electrode distance of $2 \mathrm{~cm}$. The reference electrode was placed on the anterior superior iliac spine (ASIS). Electromyography (EMG) $\mathrm{da}^{-}$ ta were collected from the following dominant side muscles: 1) rectus abdominis (RA), parallel and approximately $3 \mathrm{~cm}$ lateral and superior to the umbilicus, arranged along the longitudinal axis over the muscle belly, 2) external oblique (EO), half way between the ASIS of the pelvis and the inferior border of the rib cage at a slightly oblique angle, running parallel to the underlying muscle fibers, 3) internal oblique (IO), half way between the ASIS of the pelvic and the midline, just superior to the inguinal ligament, 4) gluteus medius (GM), over the proximal third of the distance between the iliac crest and the greater trochanter, and 5) quadratus lumborum (QL), approximately $4 \mathrm{~cm}$ lateral from the vertebra ridge and at a slightly oblique angle at half ot the distance between the 12th rib and the iliac crest. Also, EMG 
data from the non-dominant side hip adductor longus (Add) was collected at the medial aspect of the thigh in an oblique direction, $4 \mathrm{~cm}$ from the pubis. The raw data were rectified and filtered using a Lancosh FIR digital filter. A band pass filter was used between 20 and $500 \mathrm{~Hz}$ and a band stop was used at $60 \mathrm{~Hz}$. The sampling rate was $1000 \mathrm{~Hz}$. EMG data were converted to root mean square (RMS) values. For normalization, the mean RMS of three trials of five seconds of maximal voluntary isometric contractions (MVICs) was calculated for each muscles, in accordance with Kendall et al (2005). The data were expressed as a percentage of mean MVIC (\%MVIC).

\section{Three dimensional motion analysis system}

To measure pelvic lateral tilting during hip abduction in side lying, a three dimensional ultrasonic motion analysis system (CMS-HS, Zebris, Medizintechnik, Germany) was used. Three active markers were placed at the level of the dominant ASIS facing the measuring sensor. The measuring sensor was placed in front of the subject and $\mathrm{re}^{-}$ corded the ultrasonic signals from the active markers. Before the measurements were taken, the angle of the pelvic lateral tilt was set to $0^{\circ}$ in the rest position as a point of reference. Then, the relative angle of the pelvic lateral tilt during hip abduction was calculated from this reference. The sampling rate was $20 \mathrm{~Hz}$ and a low pass filter was used, with a cutoff frequency of $8 \mathrm{~Hz}$. Using the Windata software, the kinematic data were analyzed. From the three trials, the mean angle was calculated and used in data analysis.

\section{Procedures}

Subjects lay on their sides with their non dominant lower extremity in contact with a firm mattress. Before the test, the subjects were trained for 15 minutes, in order to familiarize them with PHA and CHA. PHA involves $35^{\circ}$ abduction of the dominant hip during side lying (Cynn et al, 2006). CHA involves $10^{\circ}$ adduction of the contralateral hip and $35^{\circ}$ abduction of the dominant hip during side lying. The degree of contralateral hip adduction was set at the proper angle based on a pilot study. Subjects were required to maintain steady trunk alignment during the movement without hand support. To set the degrees of abduction and adduction during the repeated test, bars were placed at the $35^{\circ}$ hip abduction and $10^{\circ}$ hip adduction positions (Figure 1). The electrodes and three markers were attached to collect EMG and kinematic data. Then, subjects were asked to perform PHA and CHA randomly according to the experimenter's orders. During the movements, muscle activity and the angle of pelvic lateral tilting were recorded at the same time. All examinations were conducted by the same experimenter.

\section{Statistical analysis}

All data was analyzed using SPSS ver. 12.0
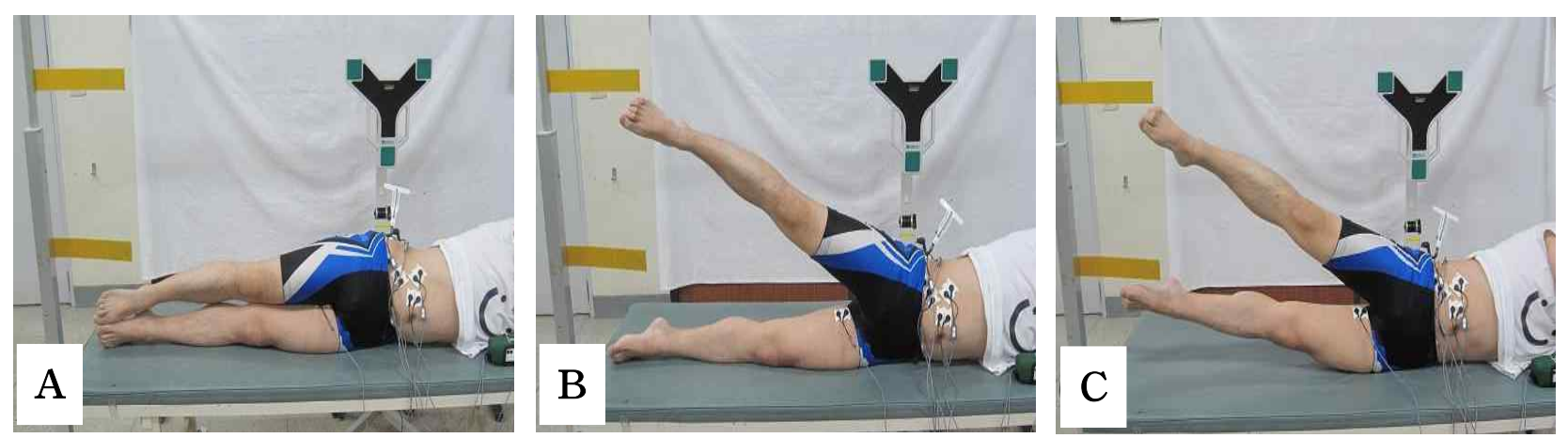

Figure 1. Test posture (A: resting position, B: preferred hip abduction, C: hip abduction with contralateral hip adduction). 
software. A paired t-test was used to determine significant differences in muscle activity of the RA, IO, EO, QL, GM and Add muscles and the pelvic lateral tilting between PHA and CHA. The level of statistical significance was set at $a=.05$

\section{Results}

\section{Muscle activity}

The EMG activity of all muscles showed significantly more increased in CHA than PHA condition $(\mathrm{p}<.05)$ (Table 2).

\section{Pelvic lateral tilting}

The angle of pelvic lateral tilting was significantly more decreased in CHA than PHA condition $(\mathrm{p}<.05)$ (Table 3).

\section{Discussion}

This study aimed to determine whether contralateral hip adduction could decrease unwanted compensatory pelvic lateral tilting during hip abduction in side lying by improving the activity of the lumbar stabilizers. It found that the activity of RA, $\mathrm{EO}, \mathrm{IO}$, and QL was significantly increased in CHA compared to PHA. These results can be explained based on several reasons.

First, the base of support (BOS) in CHA was smaller than in PHA. Subjects were asked to maintain their trunk alignment without hand support, so the contact area of the body on the floor was markedly decreased in CHA compared to PHA. This decreased BOS made the movement more challenging and led to coactive muscle contraction (Santos and Aruin, 2009). Kim et al (2011) report that the activity of abdominal muscles increased during single-leg raising in a hook laying position which provided a smaller BOS as compared with lying on the floor. The QL does not the abdominal muscles, but acts as an important lumbar stabilizer during isometric side support which produces side bending moment of the spine (McGill et al, 1996). Although the test posture in this study was different from that in past study, CHA also produced side bending moment and the test was preceded during isometric contraction. Therefore, the QL may acted as a lumbar stabilizer in this study like a previous study (McGill et al, 1996). Thus, activity of the RA, EO, IO, and QL may contract synergistically in CHA which involves a smaller BOS than PHA.

Second, the load on the lumbar vertebrae in CHA was greater than that in PHA. Cholewicki and McGill (1996) have suggested that there is a correlation between the increased relative stability index, muscle effort, and increased moment demand or the

Table 2. Comparison of the muscle activity

\begin{tabular}{ccccc}
\hline \hline Muscles & PHA $^{\mathrm{a}}$ & $\mathrm{CHA}^{\mathrm{b}}$ & $\mathrm{t}$ & $\mathrm{p}$ \\
\hline Rectus abdominis & $1.63 \pm .86^{\mathrm{c}}$ & $7.07 \pm 3.89$ & 6.29 & $<.05$ \\
External oblique & $6.17 \pm 3.89$ & $26.94 \pm 17.64$ & 5.92 & $<.05$ \\
Internal oblique & $10.46 \pm 4.77$ & $24.86 \pm 10.77$ & 7.77 & $<.05$ \\
Quadratus lumborum & $17.07 \pm 9.38$ & $54.22 \pm 24.33$ & 8.79 & $<.05$ \\
Gluteus medius & $26.11 \pm 18.38$ & $46.21 \pm 38.35$ & 4.19 & $<.05$ \\
Hip adductor longus & $1.24 \pm 2.09$ & $10.20 \pm 7.60$ & 5.48 & $<.05$ \\
\hline
\end{tabular}

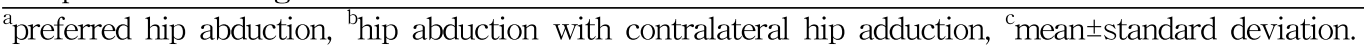

Table 3. Comparison of the angle of pelvic lateral tilting

\begin{tabular}{ccccc}
\hline \hline & PHA $^{\text {a }}$ & CHA $^{\text {b }}$ & $\mathrm{t}$ & $\mathrm{p}$ \\
\hline Pelvic lateral tilting & $11.41 \pm 4.71^{\mathrm{c}}$ & $7.78 \pm 3.19$ & 6.33 & $<.05$ \\
\hline
\end{tabular}

apreferred hip abduction, bhip abduction with contralateral hip adduction, "mean \pm standard deviation. 
joint compression force during the tasks. The activity of trunk muscles increased according to vertical and horizontal trunk load magnitude (Cholewicki et al, 2000). In addition, ten major trunk muscles (RA, $\mathrm{EO}$, IO, latissimus dorsi, iliocostalis lumborum, longissimus thoracis, lumbar erector spinae, multifidus, $\mathrm{QL})$ contribute to maintaining the stability of the lumbar spine, rather than single muscle of the trunk according to the increased load on the lumbar vertebrae (Cholewicki and Vanvliet, 2002).

Some researchers have insisted on the importance of the activity of local muscles including transversus abdominis (TrA) and the multifidus for lumbar segmental stability (Hodge and Richardson, 1997; Hodge and Richardson, 1999). Others have emphasized the importance of all of the muscles of the trunk as well as the local muscles (Cholewicki and McGill, 1996; Cholewicki and Vanvliet, 2002). The results of this study support the latter approach. Although the activity of the TrA was not addressed by this study, we could infer that $\operatorname{TrA}$ activity is increased in CHA. Marshall and Murphy (2003) have reported that the EMG activities of the TrA and IO have been shown to act together for rapid shoulder movement in all directions. Thus, activity of the TrA in CHA is also likely to be increased as is the activity of other lumbar stabilizers.

Investigating how CHA actually decreased unwanted compensatory pelvic lateral tilting, we carried out motion analysis. The results showed a significantly decreased angle of pelvic lateral tilting in CHA as compared with PHA. Kim et al (2012) have suggested that increased lumbar stabilization by isometric contraction of abdominal muscles and QL in CHA condition was effective in decreasing the angle of pelvic lateral tilting. And the activity of the GM and contralateral Add were showed significantly more increasing in CHA than PHA condition. The $\mathrm{co}^{-}$-contraction of the hip abductor and contralateral hip adductor may stabilize the pelvis in CHA (Lee, 1999), and the stabilized pelvis may decrease the angle of pelvic lateral tilting in CHA.
This study has some limitations. First, the subjects were limited to healthy young males. A study of older persons or those with injured spines may arrive at different results. Second, this was a cross-sectional study that investigated the effects of CHA on the activity of lumbar stabilizers and pelvic lateral tilting. The effects of long-term training using CHA should be examined in further studies. Third, surface EMG was used to measure muscle activity, leaving the possibility of cross-talk from adjacent muscles.

\section{Conclusion}

In this study, the effect of CHA on activity of lumbar stabilizers and compensatory movement was determined. This study found that the muscle activities of the lumbar stabilizers were significantly increased in CHA as compared with PHA. In addition, the angle of pelvic lateral tilting was decreased significantly in CHA as compared with PHA. Thus, CHA can be recommended as a hip abduction $\mathrm{ex}^{-}$ ercise for activating the lumbar stabilizers and $\mathrm{de}^{-}$ creasing compensatory pelvic lateral tilting motion.

\section{References}

Cholewicki J, McGill SM. Mechanical stability of the in vivo lumbar spine: Implications for injury and chronic low back pain. Clin Biomech (Bristol, Avon). 1996;11(1):1-15.

Cholewicki J, Simons AP, Radebold A. Effects of external trunk loads on lumbar spine stability. J biomech. 2000;33(11):1377-1385.

Cholewicki J, Vanvliet JJ 4th. Relative contribution of trunk muscles to the stability of the lumbar spine during isometric exertions. Clin Biomech (Bristol, Avon). 2002;17:99-105.

Cynn HS, Oh JS, Kwon OY, et al. Effects of lumbar stabilization using a pressure biofeedback unit 
on muscle activity and lateral pelvic tilt during hip abduction in sidelying. Arch Phys Med Rehabil. 2006;87(11):1454-1458.

Hodges PW, Richardson CA. Feedforward contraction of transversus abdominis is not influenced by the direction of arm movement. Exp Brain Res. 1997;114(2):362-370.

Hodges PW, Richardson CA. Altered trunk muscle recruitment in people with low back pain with upper limb movement at different speeds. Arch Phys Med Rehabil. 1999;80(9):1005-1012.

Hoffman M, Payne VG. The effect of proprioceptive ankle disk training on healthy subjects. J Orthop Sports Phys Ther. 1995;21(2):90-93.

Janda V. Evaluation of Muscle Imbalance. In: Liebenson $\mathrm{C}$ ed. Rehabilitation of the spine: a practitioner's manual. Baltimore, MD, Williams \& Wilkins, 1996:822.

Judge JO, Lindsey C, Underwood M, et al. Balance improvements in older women: Effects of $\mathrm{ex}^{-}$ ercise training. Phys Ther. 1993;73(4):254-262.

Jull G, Richardson C, Toppenberg R, et al. Towards a measurement of active muscle control for lumbar stabilisation. Aust J Physiother. 1993;39 (3):187-193.

Kim SJ, Kwon OY, Yi CH, et al. Comparison of abdominal muscle activity during a single-legged hold in the hook-lying position on the floor and on a round foam roll. J Athl Train. 2011;46(4): 403-408.

Kim HU, Choi BR, Kim SJ, et al. Effect of contralateral hip adduction on muscle thicknesses of lumbar stabilizers and pelvic lateral tilting during hip abduction in side-lying. Phys Ther Kor. 2012;19(1):19-27.

Kisner C, Colby LA. Therapeutic Exercise:
Foundation and techniques, 4th ed. Philadelphia, F.A. Davis Co., 2002:657-667.

Krebs DE, Elbaum L, Riley PO, et al. Exercise and gait effect on in vivo hip contact pressure. Phys Ther. 1991;71:301-309.

Lee D. The Pelvic Girdle. 2nd ed, London, Churchill Livingstone, 1999:57-60.

Marshall P, Murphy B. The validity and reliability of surface EMG to assess the neuromuscular response of the abdominal muscles to rapid limb movement. J Electromyogr Kinesiol. 2003;13(5): 477-489.

McGill S, Juker D, Kropt P. Quantitative intramuscular myoelectric activity of quadratus lumborum during a wide variety of tasks. Clin Biomech (Bristol, Avon). 1996:11(3):170-172.

Norris CM. Spinal stabilisation: 4.muscle imbalance and the low back. Physiotherapy. 1995;81(3):127138.

Root L, Spero CR. Hip adductor transfer compared with adductor tenotomy in cerebral palsy. J Bone Joint Surg Am. 1981;63(5):767-772.

Santos MJ, Aruin AS. Effects of lateral perturbations and changing stance conditions on anticipatory postural adjustment. J Electromyogr Kinesiol. 2009;19(3):532-541.

Sashika H, Matsuba Y, Watanabe Y. Home program of physical therapy: Effect on disabilities of patients with total hip arthroplasty. Arch Phys Med Rehabil. 1996;77(3):273-277.

This article was received September 29, 2013, was reviewed September 29, 2013, and was accepted October 31, 2013. 